\title{
GIH-21 Restoration of XAF1 expression inhibits gastric and colonic tumorigenesis in vivo
}

Shui Ping Tu*, Jiantao Cui, Marie C.M. Lin**, Xiao Hua Jiang*, Yi Yang*, Ching Tung Lum*, ST Yuen^, SY Leung^, Shiu Kum Lam*, Hsiang Fu Kung**, Benjamin Chun Yu Wong*. Department of Medicine*, Pathology^, and **Institute of Molecular Biology, University of Hong Kong, Hong Kong.

Introduction: XAF1 ( XIAP-Associated Factor 1 ) is identified as a novel XIAP (X-linked inhibitor of apoptosis ) binding partner. XAF1 antagonizes the anti-caspase activity of recombinant XIAP and reverses the protective effect of XIAP overexpression in cell lines. In this present study, we investigated the role of XAF1 as a tumour suppressor. Method: pcDAN3-XAF1 and Adeno-XAF1 plasmids were constructed. Adeno-XAF1 virus was generated. Stable transfectants were established and characterized. Tumourigenesis was evaluated by the formation of solid tumours in athymic nude mice xenograft.

Results: Expression of XAF1 was detected only in 50\% gastric and colon cancer cell lines, and 54\% human colon cancer tissues expressed low level of XAF1 mRNA compared with adjacent normal colon tissues. Restoration of XAF1 expression induced apoptosis in gastric and colon cancer cells. Overexpression of XAF1 suppressed anchorage-dependent and -independent growth, induced cell cycle arrest, and increased the sensitivity to chemotherapeutic drugs. Furthermore, infection of adeno-XAF1 induced apoptosis in gastric and colon cancer cells. In nude mice, overexpression of XAF1 suppressed tumorigenesis in stable gastric and colon cancer transfectants expressing XAF1. Gastric and colon cancer cells infected with adeno-XAF1 either lost their tumourigenic potential or formed tumours of smaller size and markly delayed onset when compared with those infected with adeno-LacZ and uninfected cells.

Conclusion:These results demonstrated that XAF1 is a tumour suppressor and has strong proapoptotic activity in gastric and colon cancer. XAF1 may be an ideal candidate for cancer therapy.

\section{GIH-22 Increased serum levels and epithelial expression of macrophage migration inhibitory factor in gastric cancer}

Harry H-X Xia, Xing Xiang He, Shiu Kum Lam, Yi Yang, Marie C-M Lin, Wai Man Wong, Hsiang Fu Kung, Suet Yi Leung, Siu Tsan Yuen, Benjamin C-Y Wong. Department of Medicine, Institute of Molecular Biology and Department of Pathology, The University of Hong Kong, Hong Kong

Introduction: Macrophage migration inhibitory factor (MIF) plays a pivotal role in inflammatory and immune diseases, and is also implicated in carcinogenesis. This study aimed to determine if serum levels and gastric epithelial MIF expression are associated with gastric precancerous lesions and cancer.

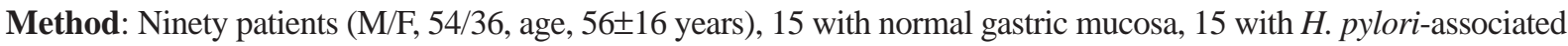
chronic antral gastritis, 20 with intestinal metaplasia in the antrum, and 40 with antral adenocarcinoma, were included in this study. Immunohistochemistry was used to determine MIF expression in gastric epithelial cells, and enzyme-linked immunosorbent assay (ELISA) was used to measure serum MIF. Five gastric cancer cell lines (AGS, MKN-45, MKN-28, MGC-803 and SGC-7901) and one non-malignant gastric cell line (GES-1) were cultured for 24 hours. MIF protein in the supernatant was measured by ELISA, and MIF mRNA in cultured cells was determined by reverse transcription-polymerase chain reaction.

Results: MIF expression in epithelial cells was weak in normal mucosa (12\%), but increased in gastritis $(52 \%)$, intestinal metaplasia $(66 \%)$ and gastric cancer $(96 \%)(\mathrm{P}<0.001$, ANOVA). Serum MIF level was also increased with the pathological changes $(576 \pm 82 \mathrm{pg} / \mathrm{ml}$ in normal mucosa, $2100 \pm 349 \mathrm{pg} / \mathrm{ml}$ in gastritis, $4498 \pm 253 \mathrm{pg} / \mathrm{ml} \mathrm{in}$ intestinal metaplasia and $9737 \pm 1249 \mathrm{pg} / \mathrm{ml}$ in gastric cancer, $\mathrm{P}<0.001$, ANOVA). There was a significant correlation between epithelial MIF expression and serum MIF level ( $\mathrm{R}=0.776, \mathrm{P}<0.001)$. Expression of MIF protein and mRNA was increased in all cancer cell lines, compared to the non-malignant cell line.

Conclusions: Advanced gastric pathology is associated with a higher MIF expression in epithelial cells. The epithelial MIF expression directly correlated with serum MIF concentrations. Hence MIF plays a role in gastric carcinogenesis and serum MIF levels may be an important biomarker for diagnosis and prognosis. 